\title{
Double Negative Left-Handed Metamaterials for Miniaturization of Rectangular Microstrip Antenna
}

\section{Ghanshyam Singh}

Department of Electronics and Communication Engineering, Jaypee University of Information Technology, Solan, India. Email: drghanshyam.singh@yahoo.com

Received May $11^{\text {th }}, 2009$; revised November 10 $0^{\text {th }}, 2009$; accepted February $20^{\text {th }}, 2010$.

\begin{abstract}
In this paper, I have explored a significant concept for the miniaturization of microstrip patch antenna configuration by using the double negative $(D N G)$ left-handed Metamaterials, which have dielectric permittivity and magnetic permeability both negative, simultaneously. It is achieved through the concept of phase-compensation by thin slab consist of the double positive (DPS) material, which have dielectric permittivity and magnetic permeability both positive, simultaneously and DNG metamaterials as a substrate of the microstrip patch antenna. By combining the DNG metamaterial slab with the slab made of DPS materials form a cavity resonator whose dispersion relation is independent of the sum of thickness of the slabs filling this cavity but it depends on the ratio of their thicknesses. This cavity constitutes by DPS and DNG material is used as substrate of the microstrip antennas and the DNG material slab is behave as phase compensator.
\end{abstract}

Keywords: Microstrip Antenna, Phase Compensation, Metamaterials, Double Negative Metamaterials

\section{Introduction}

Recent trends of the wireless mobile communication technology are towards the miniaturization and demand for more robust and compact designs have been growing [1-5]. However, in the wireless communication systems, an antenna still remains a matter of concern regarding to its size. Although, a certain level of maturity have been attained. The microstrip patch antennas, due to their inherent capabilities such as low profile, conformability, low-fabrication cost, mechanical robustness, polarization agility, compatibility/easy integration with microstrip circuits/solid state devices and adaptability to active antenna elements are widely used in all the wireless mobile communication systems. Typically, the size of a microstrip patch antenna is depends on the wavelength corresponding to its resonant frequency. There are several other factors that contribute to deciding the dimension of the antenna and its behavior such as the used substrate material and its thickness [3,4]. Literature results show that the use high-dielectric constant substrates [6], shorting walls [7], shorting pins [8,9], are effective ways for the size reduction. But the cross-polarization of such antenna is usually very high which may not be suitable for some applications [10].

The rectangular patch microstrip antenna is consists of a conductive patch on substrate materials above a conductive ground plane as shown in Figure 1. The excitation of the patch is accomplished via a microstrip feedline. This feed technique supply the electrical signal to the patch which will be converted to an electromagnetic wave. The present paper explores the possibility of miniaturization of the rectangular microstrip patch antennas using a substrate material consists of DPS and DNG metamaterials. This paper is organized as follows. The Section 2 is concern with the concept of the phase compensation. The Section 3 discusses the effects phase compensation on the size of the rectangular microstrip patch antenna and finally, Section 4 concludes the work.

\section{Phase Compensations}

Recently, there has been growing interest in the metamaterials with negative permittivity and permeability as candidate for design of the novel microwave and optical devices [1-5,11-19]. We can view metamaterials as a broader class of materials than left-handed medium. It is a class of materials that enable us to manipulate the di- 
electric permittivity and magnetic permeability. An electromagnetic wave propagating through such a material has a Poynting vector anti-parallel with its phase velocity vector as demonstrated theoretically by Veselago [17]. Such materials have been constructed in microwave range by the Smith et al. [18]. The double negative metamaterial represents a new kind of artificial dielectric media which are usually synthesized using periodic structures and exhibits the negative refractive index characteristics (resulted from simultaneous negative permittivity and permeability). Such a material have been referred to as several other names such as left handed materials, metamaterials and backward wave materials $[13,16,19]$. The DNG material unit cell employs split ring resonators and thin wires. Thin wire structures produce effective negative dielectric permittivity below the plasma frequency and the split-ring resonators can result in an effective negative permeability over a particular frequency range. Overlaying these two frequency regimes, both the permittivity and permeability are simultaneously negative thus the index of refraction may have negative real value over a pass band region. By manipulating two structures the effective permittivity and permeability can be changed separately, giving us the capability to control the position of double negative regime. Several theoretical and experimental setups have been constructed on wave reflection and refraction at an interface between a backward wave medium and positive parameter medium and radiation from traveling wave source at such interface. Engheta [14] analyzed one dimensional cavity resonator made of two cascaded planar layers of backward wave and conventional dielectric, which are placed between two perfect reflectors. Because the phase velocity in DNG slab is opposite to that in DPS layer, the DNG slab acts as phase compensator. Thus, the phase cancellation can occur, leading to resonance, when the phase changes in the two layers are equal in magnitude but opposite in direction. Thus, it turn out that the condition for resonance does not depend on the thickness of two layers but rather on their ratio. This allows one, in principle to reduce the total thickness of the resonator and will as long as the required ratio of thicknesses is maintained [13-15]. The important potential application of this concept is the antenna miniaturization.

Consider a wave traveling through two consecutive slabs as shown in Figure 2. It would experience a phase change say positive in the DPS material and a negative (opposite) phase change in the DNG material. The slab thicknesses can be adjusted to nullify the positive and negative phase shifts in the cavity formed by DPS and DNG materials so that a phase compensated resonator has to be independent of the $\lambda / 2$ constraint [3]. This property of phase-compensation demonstrates the possibility of size reduction for patch antenna on a combined
DPS-DNG substrate. There are two configurations of rectangular microstrip patch antenna with a DPS-DNG substrate have been considered.

1) The interface between the two regions is parallel to the radiating edges, and

2) The interface between the two regions is normal to the radiating edges.

For the configuration, where the DPS-DNG interface is normal to the radiating edge is has been shown through simulation that the antenna is a poor radiator $[4,14]$. The present work focuses on the case where the interface is parallel to the radiating edge. I have used the cavity model with two electric walls (PEC) and four magnetic walls (PMC) as shown in Figure 1.

The slab of conventional lossless DPS materials with positive index of refraction $n_{1}$ of thickness $d_{1}$ and another slab of the lossless DNG metamaterials with negative refractive index $-\left|\mathrm{n}_{2}\right|$ (due to double negative metamaterial) and thickness $d_{2}$. We assume that each of these slabs is impedance matched to the outside region. Let us take a monochromatic uniform plane wave normally incident on this pair of slabs as shown in Figure 2. As this wave propagates through the slab, the phase difference between the exit and entrance faces of the first slab is $\mathrm{n}_{1} \mathrm{k}_{0} \mathrm{~d}_{1}$, where $\mathrm{k}_{0}=\omega \sqrt{\varepsilon_{0} \mu_{0}}$, while the total phase difference between the front and back faces of these two layer structure is $\left|\mathrm{n}_{1}\right| \mathrm{k}_{0} \mathrm{~d}_{1}-\left|\mathrm{n}_{2}\right| \mathrm{k}_{0} \mathrm{~d}_{2}$, implying that whatever the phase difference is developed by traversing the first slab is can be compensated by traversing the second slab. If the ratio of $d_{1}$ and $d_{2}$ is chosen to be $\mathrm{d}_{1} / \mathrm{d}_{2}=\left|\mathrm{n}_{2}\right| /\left|\mathrm{n}_{1}\right|$ at the given frequency, then the total phase difference between the front and back faces of these two layer structure will become zero. This means that the DNG slab acts as phase compensator in this structure $[14,15]$. We should note that such phase compensator does not depend on the sum of the thicknesses $d_{1}+d_{2}$, rather it depend on the ratio of $d_{1}$ and $d_{2}$. Thus, in principle, $d_{1}+d_{2}$ can be any value as long as $d_{1} / d_{2}$ satisfies the above condition. Therefore, even though these two layer structure is present, the wave traversing this structure would not experience the phase difference. This feature can lead to several investing ideas in devices and component designs. This concept of a miniaturized cavity resonator can be extended to a 3-D cavity and further to a microstrip patch antenna through the cavity model. The rectangular microstrip patch antenna that has been considered in this work is placed on a DPS-DNG combined slabs as substrate as shown in Figure 1. The 1-D sub-wavelength cavity resonator' (SWCR) as it is termed consists of two slabs, one DPS and one DNG, between two PEC (perfectly conducting) walls, where the plane $\mathrm{z}=0$ is taken to be at the perfectly conducting 


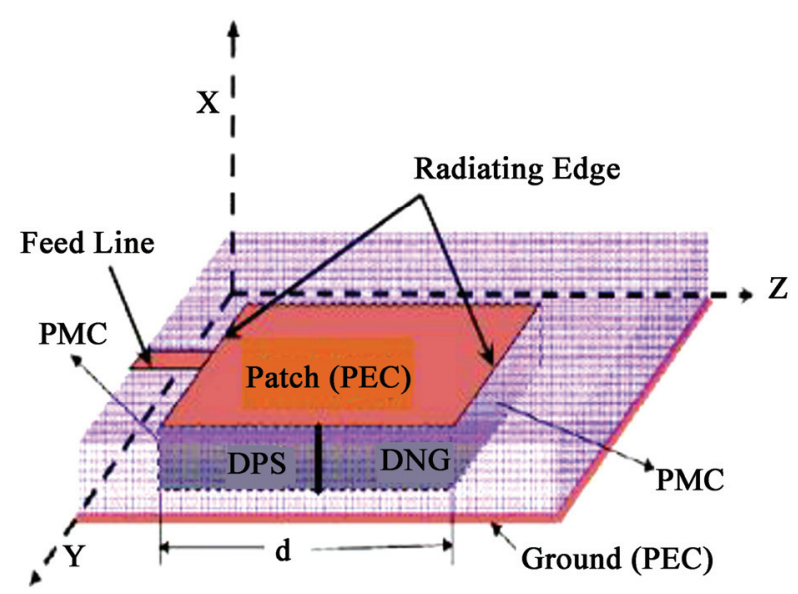

Figure 1. 3D view of DPS-DNG substrate for a rectangular microstrip patch antenna

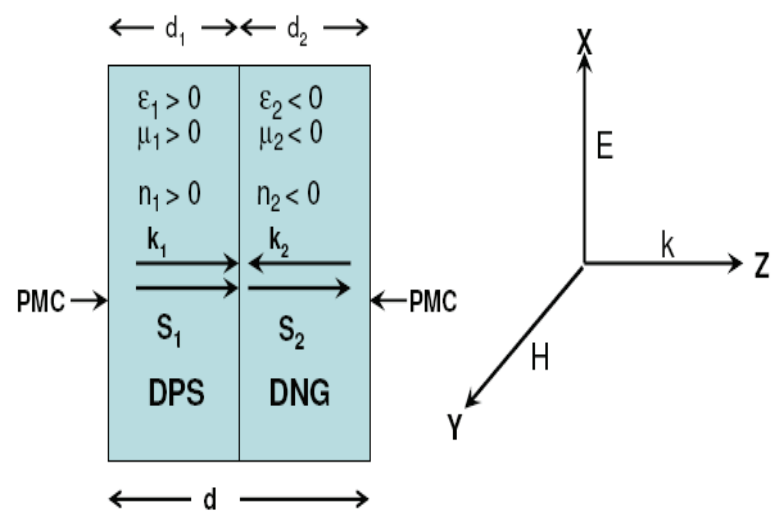

Figure 2. 1D cavity resonator made with DPS-DNG combination

plate located at the left face of the DPS. The directions of the phase velocities and the Poynting vectors in each medium are given by the arrows in the respective slabs as shown in Figure 2. The phase velocity vector in the DNG material is anti-parallel to the direction of energy flow (Poynting vector).

\section{Results and Discussion}

The idea of left-handed double-negative material as stated earlier is that when a uniform plane wave is launched in such a medium, it will have a phase velocity opposite to that of its Poynting vector. The phase compensations, in principle, are realized by combining the slabs of usual materials and a metamaterials with negative permittivity and permeability. The size of radiating edge of the patch is approximately half of the wavelength in free space for the resonant frequency of the cavity. In this configuration, the frequency dependence of the reflection coefficients from the resonator is not determined by the resonator size, but by the frequency dependence of the material parameters. This means that we deal with resonance of inclusions (split ring resonator etc.) and not by the cavity resonance (the resonator size can be further reduced if only one separate inclusion is used as resonator) as shown in Figure 3. The resonance frequency of the cavity consists of DPS and DNG materials is downshifted compared to the resonance frequency of cavity made of DPS materials. Due to this down-shift of resonance frequency, the cross-section of the radiating structure is reduced significantly. The shift in the resonant frequency to a lower value with metamaterial is promising because it suggests a reduction in the patch size as shown in Figure 3. The width of DPS slab (taken as substrate) material $d=d_{1}+d_{2}=28.33 \mathrm{~mm}$ with relative permittivity 5.9 and permeability is 1.0 . When the DPS and DNG have been taken as slab then $\mathrm{d}_{1}=17.45 \mathrm{~mm}$ and $\mathrm{d}_{2}$ $=10.89 \mathrm{~mm}$ (metamaterial), respectively.

For the simulation, I have used CST Microwave Studio which is based on finite integration technique (FIT) for general purpose electromagnetic simulator. FIT is applied to cartesian grids in the time domain is computationally equivalent to the standard finite difference time domain (FDTD) method [20]. For high frequency electromagnetic applications, time domain simulation methods are highly desirable, especially when broadband results are needed. An antenna bandwidth is mainly determined by the materials resonance response and not by the antenna size. For example, a desired resonant frequency of $10 \mathrm{GHz}$ the patch size without CSRR (complimentary split ring resonator) is $139.16 \mathrm{~mm}^{2}$ and with CSRR it is $70.85 \mathrm{~mm}^{2}$ resulting in a forty nine percent size reduction as shown in Figure 4. Certain design has a significant patch size reduction. In addition, the back lobe radiation is negligible and directivity is comparable to the traditional microstrip antennas, whose substrate is positive. It is observed that the certain designs have a significant patch size reduction and have a negligible back lobe in the radiation pattern. It shows that new kind antennas will get similar radiation patterns to the ones of conventional

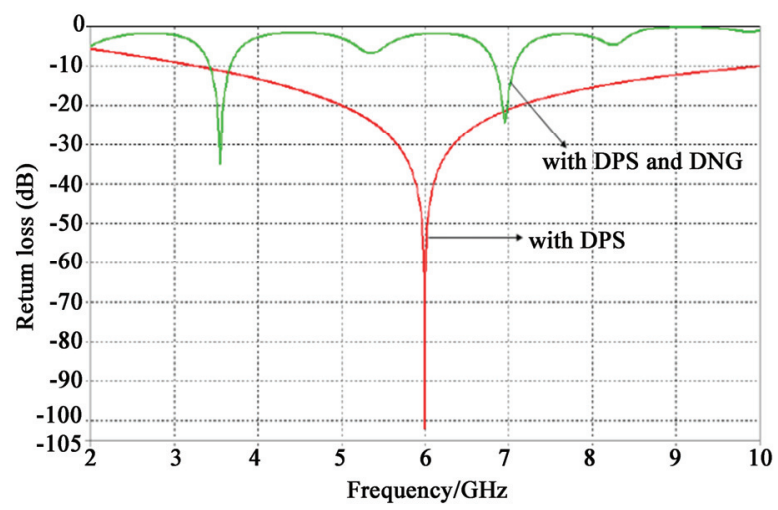

Figure 3 Frequency downshifts in the slab made by DPS and DNG 


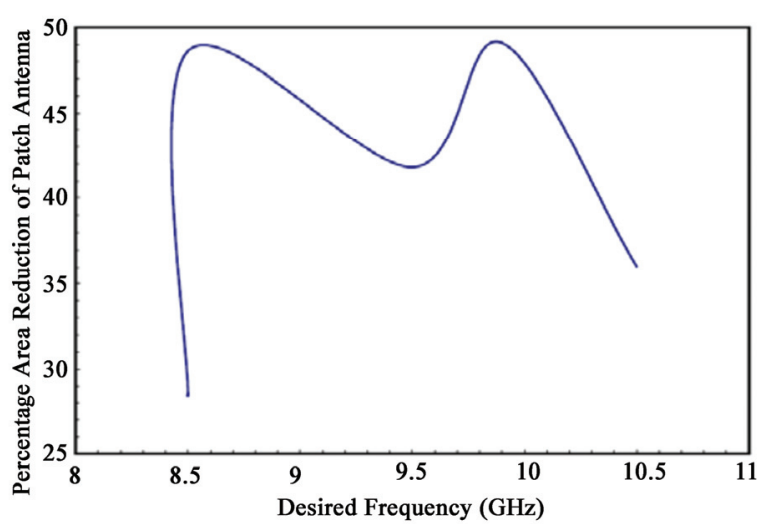

Figure 4. Percentage reduction in the size of radiating patch for a desired frequency with CSRR as compared to without CSRR in the ground plane

right-handed microstrip antennas in some case, and can yield novel radiation patterns of low elevation angles and low side lobes in other cases. These brand new radiation patterns are hardly achieved by conventional right handed microstrip antennas. By employing the substrate partially filled with double negative medium, the dimensions of the patch antenna could be significantly miniaturized, and the calculated results for the patch length 0.2 $\lambda$ show that although the use of practically dispersive double negative medium could not support such broadband performance as hypothetical non-dispersive double negative medium, but it proves the approach is perspective and important for the miniaturization of the patch antenna.

\section{Conclusions}

The size reduction of microstrip patch antennas using left-handed materials has been achieved through the concept of phase-compensation by implementing a combined DPS-DNG substrate. By employing the substrate partially filled with double negative medium, the dimension of the patch antenna could be significantly miniaturized. Many researchers are trying to improve the performance of microwave, wireless communications, microelectronics and optical devices using these new metamaterials. As the demand for small size, lightweight, and low-cost communication devices continues, the use of low-cost, small-size patch (or microstrip) antennas asserts itself. There are several variables contribute to the resonance frequency of the CSRR. An extensive study of the variable dependence will enable a designer to choose the appropriate CSRR for a particular patch antenna. A further study of radiation efficiency and bandwidth of such antennas is underway.

\section{Acknowledgements}

Author is sincerely thankful to the reviewers for the cri- tical comments and suggestions to improve the quality of the manuscript.

\section{REFERENCES}

[1] R. Yang, Y. Xie, P. Wang and L. Li, "Microstrip Antennas with Left Handed Materials Substrates," Journal of Electromagnetic Waves and Application, Vol. 20, No. 9, 2006, pp. 1221-1233.

[2] N. Engheta and R. W. Ziolkowski, "A Positive Future for Double Negative Metamaterials," IEEE Transactions on Microwave Theory and Technique, Vol. 53, No. 4, April 2005, pp. 1535-1556.

[3] M. S. Sharawi, "Use of Low-Cost Patch Antenna in Modern Wireless Technology," IEEE Potential, Vol. 25, No. 4, 2006, pp. 35-47.

[4] S. F. Mahmoud, "A New Miniaturized Annular Ring Patch Resonator Partially Loaded by a Metamaterial Ring with Negative Permeability and Permittivity," IEEE Antennas and Wireless Propagation Letters, Vol. 3, April 2004, pp. 19-22.

[5] W. Wang, B.-I. Wu, J. Pacheco, T. M. Grzegorczyk and J. A. Kong, "Metamaterial as a Substrate of Antenna Design," Progress in Electromagnetic Waves, Vol. 51, No. 10, 2003, pp. 295-338.

[6] T. K. Lo, C. O. Ho, Y. Hwang, E. K. W. Lam and B. Lee, "Miniature Aperture Coupled Microstrip Antenna of Very High Permittivity," Electronics Letters, Vol. 33, No. 1, January 1997, pp. 9-10.

[7] C. L. Mak, R. Chair, K. F. Lee, K. M. Luk and A. A. Kishk, "Half U-Slot Patch Antenna with Shunting Wall," Electronics Letters, Vol. 39, No. 25, December 2003, pp. 1779-1780.

[8] C. R. Rowell and R. D. Murch, "A Capacitively Loaded PIFA for Compact Mobile Phone Handset," IEEE Transactions on Antennas Propagation, Vol. 45, No. 5, March 1997, pp. 837-842.

[9] A. Sharma and G. Singh, "Design of Single Pin Shorted Three Dielectric Layered Substrates Rectangular Patch Microstrip Antenna for Communication Systems," Progress in Electromagnetics Research, Vol. 2, 2008, pp. 157-165.

[10] R. Chair, K. M. Lee and K. F. Lee, "Miniature Multilayer Shorted Patch Antenna," Electronics Letters, Vol. 36, No. 1, January 2000, pp. 3-4.

[11] A. Alu, F. Bilotti, N. Engheta and L. Vegni, "Subwavelength, Compact, Resonant Patch Antennas Loaded with Metamaterials," IEEE Transactions on Antennas Propagation, Vol. 55, No. 1, January 2007, pp. 13-25.

[12] H. Mosallaci and K. sarabandi, “Antenna Miniaturization and Bandwidth Enhancement Using Reactive Impedance Substrate," IEEE Transactions on Antennas Propagation, Vol. 52, No. 9, September 2004, pp. 2403-2414.

[13] S. A. Tretyakov and M. Ernutlu, "Modeling of Patch Antennas Partially Loaded with Dispersive Backward Wave Materials," IEEE Antennas and Wireless Propagation Letters, Vol. 4, No. 1, 2005, pp. 266-269. 
[14] N. Engheta, "An Idea for This Subwavelength Cavity Resonators Using Metamaterials with Negative Permittivity and Permeability," IEEE Antenna and Wireless Propagation Letters, Vol. 1, No. 1, 2002, pp. 10-13.

[15] S. Thakur and G. Singh, "Phase Compensation Using Left- Handed Double Negative Metamaterial for Compact Communication System," Proceedings of International Conference on Information and Communication Technology (IICT-2007), India, 26-28 July 2007, pp. 487-490.

[16] R. W. Ziolkowski, "Design, Fabrication and Testing of Double Negative Metamaterials," IEEE Transactions on Antennas Propagation, Vol. 51, No. 7, July 2003, pp. 1516-1529.

[17] V. G. Veselago, "The Electrodynamics of Substances with Simultaneously Negative Values of Permittivity and Permeability," Soviet Physics Uspekhi, Vol. 10, No. 4, 1968, pp. 509-514.

[18] D. R. Smith, W. J. Padilla, D. C. Vier, S. C. Nemat-Nassar and S. Schultz, "Composite Medium with Simultaneously Negative Permeability and Permittivity," Physics Review Letters, Vol. 84, No. 18, 2000, pp. 4184-4187.

[19] C. Caloz and T. Itoh, "Metamaterials for High-Frequency Electronics," Proceedings of IEEE, Vol. 93, No. 10, 2005, pp. 1144-1752.

[20] T. Weiland, 'Time Domain Electromagnetic Field Computation with Finite Difference Methods," International Journal of Numerical Modeling, Vol. 9, No. 3-4, 1996, pp. 259-319. 